\title{
Sickness certification for common mental disorders and GP return-to-work advice
}

\author{
Mark Gabbay ${ }^{1}$, Chris Shiels ${ }^{1}$ and Jim Hillage ${ }^{2}$ \\ ${ }^{1}$ Division of Health Services Research, University of Liverpool, Liverpool, UK \\ ${ }^{2}$ Institute for Employment Studies, Brighton, UK
}

\begin{abstract}
Aim: To report the types and duration of sickness certification for different common mental disorders (CMDs) and the prevalence of GP advice aimed at returning the patient to work. Background: In the United Kingdom, common mental health problems, such and depression and stress, have become the main reasons for patients requesting a sickness certificate to abstain from usual employment. Increasing attention is being paid to mental health and its impact on employability and work capacity in all parts of the welfare system. However, relatively little is known about the extent to which different mental health diagnoses impact upon sickness certification outcomes, and how the GP has used the new fit note (introduced in 2010) to support a return to work for patients with mental health diagnoses. Methods: Sickness certification data was collected from 68 UK-based general practices for a period of 12 months. Findings: The study found a large part of all sickness absence certified by GPs was due to CMDs $(29 \%$ of all sickness absence episodes). Females, younger patients and those living in deprived areas were more likely to receive a fit note for a $\mathrm{CMD}$ (compared with one for a physical health problem). The highest proportion of CMD fit notes were issued for 'stress'. However, sickness certification for depression contributed nearly half of all weeks certified for mental health problems. Only $7 \%$ of CMD fit notes included any 'may be fit' advice from the GP, with type of advice varying by mental health diagnostic category. Patients living in the most socially deprived neighbourhoods were less likely to receive 'may be fit' advice on their CMD fit notes.
\end{abstract}

Key words: common mental disorders; return-to-work advice; sickness absence; sickness certification

Received 2 September 2015; revised 20 November 2015; accepted 16 February 2016; first published online 10 March 2016

\section{Introduction}

Common mental disorders (CMDs) are widespread and contribute significantly to the global prevalence of disability (Vos et al., 2012). It has been estimated that, at any one time, one adult in six may experience symptoms of anxiety or depression. Within the workforce, a similar proportion of workers may suffer stress-related health problems (Seymour, 2010). In addition to the costs to the individual, the level of

\footnotetext{
Correspondence to: Professor Mark Gabbay, Division of Health Services Research, University of Liverpool, 1st Floor Block B, Waterhouse Building, Liverpool L69 3GE, UK. Email: mbg@liv.ac.uk
}

psychological morbidity within the working-age population can have a significant negative impact on the economy. In the United Kingdom, 160 million working days are lost a year to sickness absence, two in five due to mental health problems (Sainsbury Centre for Mental Health, 2007). Mental ill-health costs the UK economy an estimated $£ 70$ billion a year, through lost productivity, social security benefits and health service use (OECD, 2014). Mental and behavioural problems account for over $40 \%$ of claims for the main UK disability benefit, Employment and Support Allowance (DWP, 2015a). Those suffering from a mental illness are also significantly more likely to be relying on other state benefits such as income support and housing benefit (McManus et al., 2009). 
For employees with a common mental disorder the route to potential long-term work incapacity begins with the receipt of a sickness certificate from a GP. In the United Kingdom such a certificate is required by the employee after seven days of self-certification. Since 2010, this certificate has been in the form of a 'fit note', offering the GP an opportunity to advise that the patient-employee may be fit to return to work with an appropriate level of support provided by the employer. The introduction of the new certificate, replacing the long-established 'sick note', was a recognition that the patient did not have to be symptom-free to remain in work, and offered the GP a potentially key role in work retention or return-to-work interventions (Black, 2008). It has also become apparent that, similar to the prescribing of medications, the issue of a sickness certificate may have a therapeutic effect in the short-term (ie, taking a break from full-time work) but may have more negative long-term consequences (ie, worklessness). This may be particularly relevant when certificates are issued to patients with CMDs such as depression (MacDonald et al., 2012).

The level of mental-health related sickness certification does not equate to the prevalence of mild to moderate mental disorders in the working age population at large. It has been reported that for every 10 general practice consultations relating to reported depression only three resulted in a certification outcome (Mallen et al., 2011). However, in most developed countries mental disorders have replaced musculoskeletal problems as the main cause of sickness absence (Harvey et al., 2013). Empirical studies conducted in the United Kingdom have found that approximately a third of all fit notes issued by GPs are for mild to moderate mental disorders (Gabbay et al., 2015).

In the context of a changing UK welfare policy arena, where increasing attention is being paid to mental health and its impact on employability and work capacity in all parts of the welfare system, this study uses data from a national fit note database in order to focus upon three aspects of mental health-related sickness certification:

1. The likelihood of having a certified sickness episode for a CMD.

2. The prevalence of long-term certification episodes experienced by patients with different CMD diagnoses.

Primary Health Care Research \& Development 2016; 17: 437-447
3. The prevalence and types of 'may be fit' advice given by GPs issuing fit notes for different CMDs.

\section{Methods}

\section{Fit note data collection}

Fit note data for the study were generated from two projects commissioned and funded by the UK Department for Work and Pensions (DWP): the national evaluation of the fit note and the evaluation of Fit for Work Service (FFWS) pilots. The former recruited 49 general practices from five geographical areas of the United Kingdom (Scotland, Wales, Derbyshire, North West and South East England). The latter involved 19 practices sited in three FFWS pilot sites (Greater Manchester, Leicestershire and North Staffordshire). These practices varied in list size (small, medium and large), location (urban, suburban, rural) and deprivation. Although the evaluations had different objectives, both used a similar method of data collection, requesting GPs to use 'carbonised' pads of fit notes for a period of 12 months. Using the specialised pads ensured that details of every fit note issued in the period were retained on duplicate sheets. The FFWS evaluation practices started recording fit note data in July/August 2011 and the national fit note evaluation practices in November/December 2011 (Shiels et al., 2013; Gabbay et al., 2015).

In addition to the details on the note (date of issue, diagnosis, period to abstain from work, whether the patient 'may be fit' to do some work, whether the patient needed to be re-assessed at the expiry of the note and the certifying GP) a number of additional items were collected from the patient practice record that have been shown to influence sickness absence risk. These included gender, year of birth and post code. The latter was transformed by practice staff into a neighbourhood deprivation score for the patient. Deprivation scores were based on lower-level Super Output Area and Data Zone scores in the most recent Indices of Multiple Deprivation (IMD) for England, Wales and Scotland. An attempt was also made to establish whether the person receiving the fit note was normally in paid employment. However, for the majority of patients this information was not available.

A more detailed description of the data collection process has been reported previously 
(Shiels et al., 2013). Ethical approval for data collection was obtained from the (UK) National Research Ethics Service in June 2011.

\section{Data analysis}

Only patients who were recorded as being sickness absentees, that is normally in paid employment, were included in data analysis. Patients were excluded from the study if there was a record that they were not in work (and only claiming fit notes to support a benefit claim) or if there was no record of their current employment status.

Non-parametrical statistical tests $\left(\chi^{2}\right.$, Mann-Whitney) were used to test for significant differences in patient proportions and median duration of sickness episodes. Logistic regression models were run in order to test the independent effects of patient and diagnostic characteristics on likelihood of dichotomous sickness certification outcomes. Random-intercept (multilevel) models were used in the regression analysis in order to take account of the hierarchical nature of the data (patient/certifying GP/general practice). Odds ratios (ORs) and 95\% CIs are reported for each covariate.

For both univariate and multivariate analysis a conventional criterion of statistical significance $(P<0.05)$ is assumed.

Data were analysed using SPSS for Windows 22.0 and STATA IC 10.

\section{Results}

\section{The fit note database}

The fit note data collected from the two evaluations were merged by staff at the University of Liverpool, and a database was constructed. Twenty two general practices providing data had small patient list sizes ( $<5000$ registered patients). Seventeen had more than 10000 patients on their register. Forty five practices were located in an urban or suburban setting, and 23 served a largely rural population. Twenty six practices were classed as having 'highly deprived' patient populations (over $70 \%$ of their patients living in the $20 \%$ most deprived neighbourhoods in the country) and 24 were estimated to be 'low deprivation' practices ( $<20 \%$ of patients in the most deprived areas). The median weighted IMD score for the 50 English practices in the study was 20.3, approximating the median deprivation score (22.1) for all 8231 practices registered in England in 2012.

The 68 practices submitted details of 79815 fit notes issued to 33768 patients in the data collection period. A total of 42402 discrete certified sickness absence 'episodes' (containing one or more continuous fit notes) were identified. However, for only 10969 patients (32\% of patients in database), 13694 certified sickness episodes and 25078 fit notes was there a record that the patient receiving the fit note (s) was normally in paid employment. There were no statistically differences in patient characteristics (gender, age, social deprivation) between the recorded employment group and the remainder of patients (recorded as 'not in work' or with no record of employment status) that were excluded from subsequent analysis. Nearly 29\% (3950/13694) of episodes were for a CMD. The likelihood of having an episode of certified sickness absence for a mental health reason (rather than for a physical health problem) was associated with female gender, younger age and higher social deprivation. A higher proportion of female patients had at least one mental health related episode $(34 \%(2174 / 6461))$, compared to $26 \%(1190 / 4508)$ of males, $P<0.001)$. Nearly $34 \%(1925 / 5752)$ of patients below the age of 45 had a mental health-related episode, compared with $28 \%(1436 / 5201)$ of the older patients $(P<0.001)$. Nearly 33\% $(968 / 2999)$ of patients living in the $20 \%$ most deprived neighbourhoods of their country had a CMD episode, compared with $30 \%(2318 / 7701)$ of those residing in less deprived areas $(P=0.02)$. When the three patient variables (gender, age, social deprivation) were entered as covariates in a logistic regression model, they all retained a significant effect on likelihood of a CMD outcome: female, OR $=1.41$ (95\% CI 1.30-1.54); aged under $45, \mathrm{OR}=1.30$ (95\% CI 1.20-1.41); most deprived, OR = 1.09 (95\% CI 1.01-1.20).

\section{Fit notes for different mental health problems}

A total of 27792 fit notes in the database were issued to patients presenting with a CMD as the reason for sickness absence. However, a definitive record that the CMD patient receiving the fit note was normally in paid employment was only available for 3288 patients receiving a total of 8074 fit notes.

Table 1 reports the number and proportion of these 8074 fit notes within mental health diagnostic categories. Fit notes to certify sickness absence Primary Health Care Research \& Development 2016; 17: 437-447 
Table 1 Number of fit notes by CMD category

\begin{tabular}{lcccc}
\hline Column percentages & $\begin{array}{l}\text { No of fit } \\
\text { notes }\end{array}$ & $\begin{array}{l}\text { \% of all CMD fit notes } \\
\text { issued }\end{array}$ & $\begin{array}{l}\text { No of weeks certified by } \\
\text { notes }\end{array}$ & $\begin{array}{l}\text { \% of all CMD weeks } \\
\text { certified }\end{array}$ \\
\hline Stress & 2929 & 36.3 & 7942 & 30.5 \\
Depression & 2528 & 31.3 & 9490 & 36.4 \\
Anxiety & 1048 & 13.0 & 3276 & 12.6 \\
Depression (with anxiety) & 703 & 8.7 & 2797 & 10.7 \\
Bereavement reaction & 577 & 7.1 & 1357 & 5.3 \\
Substance misuse & 96 & 1.2 & 530 & 2.0 \\
Other CMD & 193 & 2.4 & 652 & 2.5 \\
All CMDs & 8074 & 100 & 26044 & 100
\end{tabular}

$\mathrm{CMD}=$ common mental disorders.

due to stress were the most prevalent, contributing over $36 \%$ of the total notes issued for CMDs. However, sickness certificates issued for depression, either alone or mixed with anxiety symptoms, accounted for over $47 \%$ of all weeks certified by the CMD fit notes.

For the group of 3288 patients that received a fit note for a CMD, there were gender and age differences in the type of note they received. A significantly higher proportion of male patients had at least one fit note for depression (36\% (399/1124) versus $31 \%(677 / 2164)$ of females, $P=0.02)$ and substance misuse $(3 \%(28 / 1124)$ versus $1 \%(14 /$ 2164) of females, $P<0.001)$. However, a significantly higher proportion of female patients received certified sickness absence for a bereavement $(11 \%(246 / 2164)$ versus 8\% (95/1124) of males, $P=0.009$ ). Younger patients (aged under $45)$ were more likely than older patients to have had a fit note for depression (35\% 661/1897) versus $29 \%$ (414/1388), $P=0.002)$ or depression with anxiety (12\% (223/1897) versus 9\% (132/1388), $P=0.04)$. However a higher proportion of patients in the older group received a fit note for a bereavement $(15 \%$ (198/1388) versus 8\% (142/1897), $P<0.001)$. No significant differences were found between the group of patients living in the $20 \%$ most deprived neighbourhoods in their country $(n=919)$ and the less deprived patients, in terms of the diagnostic category of CMD fit notes they received.

\section{Type and duration of mental health-related episodes}

From the $8074 \mathrm{CMD}$ fit notes issued to working patients, a total of 3950 discrete continuous episodes of certified sickness were identified. Nearly $48 \%(n=1878)$ of episodes consisted of more than one fit note. Fifteen per cent (492/3288) of patients had more than one mental healthrelated episode in the data collection period. Nearly $39 \%$ of episodes were for stress, $28 \%$ for depression, $13 \%$ for anxiety, $8 \%$ for mixed anxiety and depression, $8 \%$ for bereavement and just over $1 \%$ for substance misuse.

In terms of duration of CMD episodes, $38 \%$ lasted less than three weeks, $29 \%$ between three and six weeks, $17 \%$ between 6 and 12 weeks and $16 \%$ were considered 'long-term' (at 12 weeks or more; Table 2). The majority of the episodes for bereavement and $46 \%$ of those for stress were relatively short-term, lasting under three weeks in duration. Sickness episodes certified because of patient substance misuse were relatively few in number $(n=55)$ but nearly $42 \%$ reached the 12 week long-term threshold. Similar proportions of depression episodes and those for depression mixed with anxiety lasted 12 weeks or more (21\%). Depression (alone or with anxiety) accounted for $47 \%(294 / 619)$ of all long-term CMD episodes.

A significantly higher proportion of the older age-group ( $\geqslant 45$ years of age) of patients had a long-term certified episode for a CMD (20\% $(280 / 1383)$ versus $15 \% \quad(284 / 1896), \quad P<0.001)$. There were no significant associations between having an episode of 12 weeks and patient gender or social deprivation of residential area.

When the three patient variables (gender, agegroup and social deprivation) and the seven CMD categories were entered as covariates into a logistic regression model, patient age retained the 
Table 2 Duration of CMD certified sickness episodes

\begin{tabular}{|c|c|c|c|c|c|}
\hline \multirow[t]{2}{*}{ Row percentages } & \multirow[t]{2}{*}{ Total episodes } & \multicolumn{4}{|c|}{ Duration of episode } \\
\hline & & $\begin{array}{l}\text { Less than three } \\
\text { weeks }[n(\%)]\end{array}$ & $\begin{array}{l}\text { Three to } \\
\text { six weeks }[n(\%)]\end{array}$ & $\begin{array}{l}\text { More than six weeks, } \\
\text { less than } 12 \text { weeks }[n(\%)]\end{array}$ & $\begin{array}{l}12 \text { weeks } \\
\text { or more }[n(\%)]\end{array}$ \\
\hline Stress & 1533 & $705(46.0)$ & $417(27.2)$ & $226(14.8)$ & $185(12.1)$ \\
\hline Depression & 1107 & $294(26.7)$ & $345(31.3)$ & $236(21.4)$ & $228(20.7)$ \\
\hline Depression (with anxiety) & 317 & $76(24.0)$ & $118(37.2)$ & $57(18.0)$ & $66(20.8)$ \\
\hline Anxiety & 505 & $201(39.8)$ & $133(26.3)$ & $83(16.4)$ & $88(17.4)$ \\
\hline Substance misuse & 55 & $10(18.2)$ & $10(18.2)$ & $12(21.8)$ & $23(41.8)$ \\
\hline Bereavement reaction & 315 & $174(55.2)$ & $90(28.6)$ & $37(11.7)$ & $14(4.4)$ \\
\hline Other CMD & 122 & $47(39.5)$ & $40(33.6)$ & $17(14.3)$ & $15(12.6)$ \\
\hline All CMDs & 3950 & 1507 (38.2) & $1153(29.3)$ & $668(17.0)$ & $619(15.7)$ \\
\hline
\end{tabular}

$\mathrm{CMD}=$ common mental disorders.

Table 3 CMD fit notes and certified episodes with 'may be fit for work' advice

\begin{tabular}{llccc}
\hline $\begin{array}{l}\text { Row } \\
\text { percentages }\end{array}$ & $\begin{array}{l}n \text { of fit notes in } \\
\text { CMD category }\end{array}$ & $\begin{array}{l}n(\%) \text { of fit notes with } \\
\text { 'may be fit' advice }\end{array}$ & $\begin{array}{l}n \text { of certified episodes } \\
\text { in CMD category }\end{array}$ & $\begin{array}{l}n \text { (\%) of episodes completed } \\
\text { with a 'may be fit' note }\end{array}$ \\
\hline $\begin{array}{l}\text { Stress } \\
\text { Depression }\end{array}$ & 2929 & $191(6.5)$ & 1533 & $157(10.2)$ \\
Anxiety & 1048 & $179(7.1)$ & 1103 & $119(10.8)$ \\
$\begin{array}{l}\text { Depression } \\
\text { (with anxiety) }\end{array}$ & 703 & $77(7.3)$ & 505 & $50(9.9)$ \\
$\begin{array}{l}\text { Bereavement } \\
\text { reaction }\end{array}$ & 577 & $60(8.5)$ & 317 & $32(10.1)$ \\
$\begin{array}{c}\text { Substance } \\
\text { misuse }\end{array}$ & 96 & $45(7.8)$ & 315 & $24(7.6)$ \\
$\begin{array}{l}\text { Other CMD } \\
\text { All CMDs }\end{array}$ & 193 & $2(2.1)$ & 55 & $1(1.8)$ \\
\hline
\end{tabular}

independent significant association with the 12 week episode outcome: age 45 and over, $\mathrm{OR}=1.64$ (95\% CI 1.35-1.98). Compared with the stress reference category, patients in other major categories had a significantly raised risk of the 12 week outcome: substance misuse, $\mathrm{OR}=5.90 \quad(95 \% \quad$ CI $2.97-11.7) ;$ depression, $\mathrm{OR}=2.32$ (95\% CI 1.86-2.86); depression (with anxiety), OR $=2.30$ (95\% CI 1.68-3.16); anxiety, $\mathrm{OR}=1.67$ (95\% CI 1.26-2.22).

\section{'May be fit' advice in mental health episodes}

The format of the fit note provides an opportunity for the GP to advise that the patient may be able to return to work (even if not fully symptomfree) provided there is an appropriate source of support. Structured options of support listed on the fit note are 'amended duties', 'altered hours, 'phased return' and 'workplace adaptations' The GP can check one or more of these options, and/or add free-text in an 'additional comments' section.

Seven per cent of CMD fit notes included 'may be fit' advice from the certifying doctor. The rate of issuing this type of note was similar across the depression, stress and anxiety categories (Table 3 ). Nearly $10 \%$ of certified sickness absence episodes had 'may be fit' advice on the final fit note, thus completing the episode. These 'may be fit' episodes were longer than those episodes where no return to work advice was offered (median weeks 5.5 weeks compared with 4 weeks, $P<0.001$ ). Longer duration of these 'may be fit' episodes was found for stress (5.4 versus 3.0 weeks, $P<0.001)$, depression ( 5.8 versus 4.4 weeks, $P=0.38$ ), anxiety (5.8 versus 4.0 weeks, $P=0.08$ ), bereavement

Primary Health Care Research \& Development 2016; 17: 437-447 
Table 4 Types of 'may be fit' advice on fit notes issued to CMD patients

\begin{tabular}{|c|c|c|c|c|c|c|}
\hline \multirow{2}{*}{$\begin{array}{l}\text { Row } \\
\text { percentages }\end{array}$} & \multicolumn{6}{|c|}{ Structured advice item checked on fit note } \\
\hline & $\begin{array}{l}\text { No of 'may } \\
\text { be fit' notes }\end{array}$ & $\begin{array}{l}\text { 'Phased return to } \\
\text { work' }[n(\%)]\end{array}$ & $\begin{array}{l}\text { 'Altered hours of } \\
\text { work' }[n(\%)]\end{array}$ & $\begin{array}{l}\text { 'Amended work } \\
\text { duties' }[n(\%)]\end{array}$ & $\begin{array}{l}\text { 'Workplace } \\
\text { adaptations' } \\
{[n(\%)]}\end{array}$ & $\begin{array}{l}\text { Comment (written } \\
\text { advice) }[n(\%)]\end{array}$ \\
\hline Stress & 191 & $90(47.1)$ & $61(31.9)$ & $53(27.7)$ & $18(9.4)$ & $115(60.2)$ \\
\hline Depression & 179 & $112(62.6)$ & $61(34.1)$ & $33(18.4)$ & $8(4.5)$ & $100(55.9)$ \\
\hline Anxiety & 77 & $47(61.0)$ & $18(23.4)$ & $23(29.9)$ & $8(10.4)$ & $38(49.4)$ \\
\hline $\begin{array}{l}\text { Depression } \\
\text { (with anxiety) }\end{array}$ & 60 & $36(60.0)$ & $19(31.7)$ & $11(18.3)$ & $5(8.3)$ & $39(65.0)$ \\
\hline Bereavement & 45 & $28(62.2)$ & $7(15.6)$ & $7(15.6)$ & $3(6.7)$ & $22(48.9)$ \\
\hline All CMDs & 562 & $318(56.6)$ & $169(30.1)$ & $129(23.0)$ & $43(7.7)$ & $321(57.1)$ \\
\hline
\end{tabular}

Row percentages total $>100$.

$\mathrm{CMD}=$ common mental disorders.

(4.2 versus 2.1 weeks, $P<0.001)$ and depression with anxiety ( 4.2 versus 4.1 weeks, $P=0.98$ ).

No significant associations were found between either the patient's gender or age, and the likelihood of receiving any 'may be fit' notes for a CMD during the period of data collection. However, a significantly lower proportion of patients living in the $20 \%$ most deprived neighbourhoods in the country had received a "may be fit' note (11\% (105/919) compared with 14\% (318/ 2292) of those living in less socially deprived areas, $P=0.04)$.

For the $562 \mathrm{CMD}$ fit notes that did include 'may be fit' advice the most common recommendation for adjustment to facilitate a return to work was for the patient to be allowed to phase a return to work (Table 4). This option was indicated by the GP on nearly $57 \%$ of all 'may be fit' notes, either alone or with other advice. For depression fit notes, the proportion was higher at nearly $63 \%$. Over $30 \%$ of all CMD 'may be fit' notes included GP advice that the patient's normal hours of work should be altered in order to enable a prompter return to work. This type of advice was more prevalent on depression fit notes (over $34 \%$ including this advice).

Twenty three per cent of 'may be fit' notes recommended that the return to work might be facilitated by amending the patient's normal work duties. This advice was more likely to be found on stress $(28 \%)$ and anxiety $(30 \%)$ notes rather than those issued for depression (18\%). The recommendation for workplace adaptations to be made by the employer to enable the patient to end their sickness absence was least prevalent (found on nearly $8 \%$ of all 'may be fit' notes). Over $57 \%$ of notes included a written comment from the GP, usually clarifying the advice indicated by a structured option.

\section{Discussion}

\section{Summary of main findings}

The study found a large part of all sickness absence certified by GPs was due to common mental disorders $(29 \%$ of sickness absence episodes). This mental health-related sickness certification was more prevalent among females, the younger group of patients and those living in the most socially deprived neighbourhoods. In terms of different mental health diagnoses, fit notes for stress were the most prevalent, but depression (alone or with anxiety symptoms) had the largest impact on the total duration of CMD sickness absence ( $47 \%$ of all weeks certified by CMD fit notes).

Nearly $16 \%$ of all CMD episodes were classed as 'long-term' (lasting 12 weeks or more). However, distribution of episode duration varied across diagnostic categories. The majority of episodes for bereavement and nearly half of stress episodes were 'short-term' (less than three weeks in length). Older patients were more likely to have a longterm episode (for any CMD). Substance misuse (although contributing relatively few fit notes and episodes) was most likely to result in a long-term episode, with depression also an important diagnostic predictor of a 12 week episode. 
Only 7\% of CMD fit notes had the 'may be fit' section completed, whereby the GP could recommend a return to work provided there was appropriate support available or adjustments made. Patients living in the most deprived neighbourhoods were less likely to have received this type of note for their CMD. The most common items of advice indicated by GPs were for the patient to be allowed to phase a return to work ( $57 \%$ of all 'may be fit' notes) and for the employer to allow the patient to alter normal working hours (30\%). Advice relating to an amendment of the patient's normal work duties $(23 \%$ of all 'may be fit' notes) was more prevalent on sickness certificates issued for anxiety or stress (rather than depression).

\section{Strengths and weaknesses of study}

The main strength of the study is its use of data extracted from the University of Liverpool fit note database, the largest collection of routine sickness certification data in the UK. The 68 general practices providing details were a representative national sample in terms of geographical location, patient list size and social deprivation of practice catchment areas. An important limitation of the study is the dependence on the patient general practice medical record as the source of potential explanatory data items. This restricted patient characteristics to gender, age and social deprivation (derived from patient post code of residence). No other patient data is routinely and consistently recorded at general practices in the United Kingdom. The study would have been strengthened by the availability of employment and occupational data.

\section{Relation to previous research}

Similar proportions of mental health-related sickness certification were found in previous studies utilising all or part of the same fit note database (Shiels et al., 2013; Gabbay et al., 2015). While in advanced industrial societies musculoskeletal health problems have long been the major cause of work incapacity, in recent years the importance of CMDs as a proportion of all sickness absence has increased (Martin et al., 2009). In countries with a large manufacturing sector, such as those in Eastern Europe (Poland, Slovakia), physical health problems still dominate claims for disability benefits. However in other countries, with a relatively large service sector, mental health problems have become the primary cause of long-term incapacity (EFILWC, 2007). In the United Kingdom there is some evidence that the proportion of CMD sickness certification has increased considerably in the last decade (Gabbay et al., 2015).

Of all the various mental health diagnoses, this study found that diagnoses of depression made the greatest contribution to the total period of certified sickness absence. This relative importance of depression vis-à-vis other CMDs has been reflected in its predominant use in previous studies focusing on association between sickness absence and mental health disorders (Knudsen et al., 2013). Anxiety, despite having potentially similar negative functional outcomes, has been rarely used as the mental health problem of interest in sickness absence research (Laitinen-Krispijn and Bijl, 2000; Knudsen et al., 2013).

Our definition of a 'long-term' episode is based largely on post-hoc analysis of distribution of episode duration in the fit note database. The 12 week threshold was identified as a key point in the path to long-term work incapacity. While the definition of 'long-term' varies between studies (often linked to the social insurance system in the respective country of the study) some important Scandinavian studies have used a similar 90-day threshold in focusing on the impact of mental health on long-term sickness absence (Hensing et al., 1996; Vaez et al., 2007; Knudsen et al., 2013). The influential Hordaland Health Study found that nearly a quarter of mental health sickness episodes were for longer than 90 days. Anxiety and mixed anxiety with depression were significant factors for sickness absence in general, but depression was a major predictor of the longer episodes (Knudsen et al., 2013).

\section{Implications for policy and practice}

It was anticipated that the introduction of the fit note in 2010 would encourage GPs to have a more active role in supporting sickness absentees back to work. In particular it was hoped that the new sickness certificate would make it easier for the GP to initiate discussion with the patient about a possible return to work, and would result in an improved level of communication between the GP, employer and employee (Black, 2008). Qualitative research evidence suggests that it may indeed have 
improved the levels of discussion and communication (Chenery, 2013). However, research aimed at eliciting GP views of the new sickness certificate has reported perceived difficulties in completing the 'may be fit' options, a reluctance to compromise the GP-patient relationship by advising a return to work and dissatisfaction with the lack of feedback provided by the employer on the efficacy of the GP advice on the fit note (Hann and Sibbald, 2013). Evaluation of the fit note in practice suggests that the opportunity for the GP to recommend that the patient may be fit to return to work, with appropriate support, has not been utilised to the optimal degree. This lack of 'may be fit' advice is particularly evident in the case of sickness absentees presenting with mental health problems (Shiels et al., 2014).

The need for the GP to play a more active role in interventions is even more urgent when we consider that the vast majority of people with CMDs have their first consultation in general practice (Gask et al., 2009). A recent survey found that most GPs have identified the large upward trend in the presentation of mental health problems such as anxiety and stress by their patients in the past few years (Aviva, 2013). In fact, the relatively low proportion of 'may be fit' notes issued to mental health patients may be associated with a wider problem within the primary care sector; a shortage of skills in the diagnosis and treatment of people with a CMD. It has been reported that GPs are more likely to treat CMDs with anti-depressants alone rather than recommend evidence-based treatments that have been proven to support a return to work (OECD, 2014). There is some evidence that GPs trained to diagnose and treat depression can help people to retain employment (Simon et al., 1998; Schoenbaum et al., 2001; 2002; Rost et al., 2004; Lo Sasso et al., 2006). It may also be true that, at present, many GPs lack knowledge about the demands of workplaces, the impact on mental health of various working environments, and the appropriate adjustments to the patient's working conditions required for the person with a CMD to return to work (OECD, 2014). Of course, if the GP does recommend that the patient may be able to work if some adjustments are made, the employer can either comply with or disregard the advice on the fit note. So if long-term CMD patients are not returning to work in numbers, the employer has to accept a share of the responsibility. Previous research has suggested that employers may be more willing to make the necessary adjustments for employees returning from long-term sickness absence for a physical health problem rather than those who might be able to return after suffering a $\mathrm{CMD}$, seeing the latter as having problems that were more likely to be intertwined with work-based issues (Mental Health Foundation, 2009).

The 2011 independent review of sickness absence in Great Britain recommended that GPs should be able to refer patients who have been off work for a relatively short period (four weeks or more) to a newly established assessment and advisory service that would provide the patient with the guidance and support required to enable a return to work (Black and Frost, 2011). The new service, Fit for Work (FfW), is currently being rolled out to all general practices in England and Wales, with it being anticipated that all practices should be able to refer patients to FfW by late 2015 . Referral will only be considered if the patient has been off work for four weeks (including the first seven days of selfcertified sickness), the GP feels that the patient has reasonable prospects of returning to work and the patient consents to referral. It is anticipated that the successful operation of the service will result in a number of benefits to GPs and their patients. These include GP access to a specialist occupational health resource offering independent quality advice, support for the GP in managing difficult return-to-work consultations and an easy-to-use rapid intervention that will support health outcomes for the patient (DWP, 2015c). For employers, FfW may potentially reduce sick pay costs and provide small and medium-sized enterprises with much needed access to occupational health advice and services (DWP, 2015b)

When fully operational, the FfW should have the potential to significantly reduce the burden of longterm certified sickness absence via the opportunities for early intervention and integrating occupational health and employment functions. However, given the very high proportion of CMD-related episodes of sickness absence (reported in ours and earlier studies) the service will need to take into account the specialised needs of those patients referred with mental health issues when drawing up return-towork plans. Given the fact that limited consultation time makes it difficult for the GP to conduct a formal psychological assessment of the patient, it will be important that the new service has the capacity for appropriate assessment using valid measures, 
and to provide integrated work-related and psychological services. This raises the issue of how the service can build the necessary capacity, in terms of a trained workforce, to meet a potentially large demand. We found that over $60 \%$ of CMD episodes met the eligibility criterion of four weeks sickness absence (ie, three weeks of sickness certification). While it is likely that a significant proportion of these patients would not be suitable for referral or would not consent, and that the majority of referrals may be able to receive a telephone assessment, there is still a potential for the FfW service to be overwhelmed. However, the alternative outcome is also possible, and the lack of awareness of the service will lead to smaller numbers of referrals than was anticipated. A survey of 1000 GPs in March 2015 found that $61 \%$ were not aware of the new service, and that two-thirds of those who were did not intend to refer patients, believing that employers had the main responsibility for dealing with sickness absence (Cigna and YouGov, 2015). In the case of mental health, there is also the concern that the barriers that have prevented the GP from including 'may be fit' advice on CMD fit notes will also lead to mental health patients who might have benefited from the new service not being referred.

Given the potential resource constraints faced by the FfW, and the existing lack of return-to-work advice included in fit notes issued to CMD patients, it may be that GPs have to focus on specific groups of patients in order to maximise the efficacy of referral and any 'may be fit' advice. Based on some of the key findings of this study, three groups of CMD patients might be targeted by the GP. First, older patients, although less likely to receive fit notes for a CMD, are more likely than younger patients to have a long-term episode. Recovery may be slower when a CMD occurs later in life, and these patients will need extra support to prevent a potential exit from the labour force altogether. Second, patients living in socially deprived neighbourhoods are more likely than those in less deprived areas to be issued a fit note for a CMD, but are less likely to receive 'may be fit' advice in their fit notes. While the reasons for this are unclear, GPs working with deprived patients need to be aware of this anomaly, and respond accordingly. Thirdly, patients with depression are more likely than those with other common mental health problems to go on to have a long period of sickness certification. The core symptoms of depression affect the employee's emotional, cognitive and social functioning, which directly impacts on their ability to perform normal work duties (Harvey et al., 2013). However, for patients with no history of previous depression, and with less severe symptoms, early intervention is important in order to prevent transition to long-term work incapacity. Early referral to the FfW and/or appropriate 'may be fit' advice may facilitate a return to work, without the need for a GP referral to a psychological health service.

\section{Conclusions}

Common mental disorders are the major diagnostic cause of long-term work incapacity and claims for incapacity-related benefits in the United Kingdom. However, it needs to be recognised that different mental health diagnoses (such as depression and stress) have potentially diverse implications for sickness certification outcomes (including the appropriate return-to-work advice). The GP may have a key role to play in strategies to reduce the numbers of patients with mental health problems who have long-term sickness certification and (if not addressed) may eventually leave the workforce for good. In particular, more effective use of the 'may be fit' section on CMD fit notes and appropriate referral to the newly established Fit for Work service may both be ways in which the GP can help their patients to return to (and retain) their usual employment.

\section{Acknowledgements}

We are grateful to the clinical and administrative staff involved in the collection of data at the 68 general practices. Professor Gabbay is part funded by (NIHR-National Institute for Health Research) CLAHRC-NWC.

\section{Financial Support}

The collection of sickness certification data was funded by the (UK) Department for Work and Pensions.

\section{Conflicts of Interest}

None.

Primary Health Care Research \& Development 2016; 17: 437-447 


\section{Ethical Standards}

The authors assert that all procedures contributing to this work comply with the ethical standards of relevant national and institutional guidelines and with the Helsinki Declaration of 1974, as revised in 2008.

\section{References}

Aviva. 2013: The Aviva Health of the Nation index. February 2013.

Black, C. 2008: Working for a healthier tomorrow. London: The Stationery Office.

Black, C. and Frost, D. 2011: Health at work: an independent review of sickness absence. London: The Stationery Office.

Chenery, V. 2013: An evaluation of the statement of fitness for work (Fit note): a survey of employees. DWP research report no 840. London: The Stationery Office.

Cigna and YouGov. 2015: DWP Fit for Work awareness research.

Department for Work and Pensions (DWP). 2015a: Employment and support allowance: outcomes of work capability assessment. Quarterly Statistics. Retrieved 15 March 2015 from https://www. gov.uk/government/uploads/system/uploads/attachment_data/ file/411937/esa_wca_summary_Mar15_final.pdf.

Department for Work and Pensions (DWP). 2015b: Fit for work: guidance for employers. Retrieved 2 August 2015 from https://www.gov.uk/government/organisations/ department-for-work-pensions.

Department for Work and Pensions (DWP). 2015c: Fit for work: guidance for GPs. Retrieved 2 August 2015 from https://www.gov.uk/government/organisations/departmentfor-work-pensions.

European Foundation for Improvement of Living and Working Conditions (EFILWC). 2007: Fourth European work conditions survey. Luxembourg: Office for Publications of EU.

Gabbay, M., Shiels, C. and Hillage, J. 2015: Factors associated with the length of fit note-certified sickness episodes in the UK. Occupational and Environmental Medicine 72, 467-75.

Gask, L., Lester, H. and Kendrick, T. 2009: Primary care mental health. London: Royal College of Psychiatrists.

Hann, M. and Sibbald, B. 2013: General practitioners' attitudes towards patients' health and work, 2010-12. DWP research report no 835. London: The Stationery Office.

Harvey, S.B., Joyce, S., Modini, M., Christensen, H., Bryant, R., Mykletun, A. and Mitchell, P.B. 2013: Work and depression/ anxiety disorders - a systematic review of reviews: final report. Melbourne, Australia: Beyondblue.

Hensing, G., Alexanderson, K., Allebeck, P. and Bjurulf, P. 1996: Sick leave due to psychiatric disorder: higher incidence among women and longer duration for men. British Journal of Psychiatry 169, 740-46.

Knudsen, A.K., Harvey, S.B., Mykletun, A. and Øverland, S. 2013: Common mental disorders and long-term sickness

Primary Health Care Research \& Development 2016; 17: 437-447 absence in a general working population. The Hordaland Health Study. Acta Psychiatrica Scandinavica 127, 287-97.

Laitinen-Krispijn, S. and Bijl, R.V. 2000: Mental disorders and employee sickness absence: the NEMESIS study. Social Psychiatry and Psychiatric Epidemiology 35, 71-77.

Lo Sasso, A.T., Rost, K. and Beck, A. 2006: Modeling the impact of enhanced depression treatment on workplace functioning and costs: a cost-benefit approach. Medical Care 44, 352-58.

MacDonald, S., Maxwell, M., Wilson, P., Smith, M., Whittaker, W., Sutton, M. and Morrison, J. 2012: A powerful intervention: general practitioners' use of sickness certification in depression. BMC Family Practice 13, 82.

Mallen, C., Wynne-Jones, G. and Dunn, K. 2011: Sickness certification for mental health problems: an analysis of a general practice consultation database. Primary Health Care Research and Development 12 (2), 179-82.

Martin, A., Sanderson, K. and Cocker, F. 2009: Meta-analysis of the effects of health promotion intervention in the workplace on depression and anxiety symptoms. Scandinavian Journal of Work, Environment \& Health 35, 7-18.

McManus, S., Meltzer, H., Brugha, T., Bebbington, P. and Jenkins, R. 2009: Adult psychiatric morbidity in England, 2007: results of a household survey. London: National Centre for Social Research.

Mental Health Foundation. 2009: Returning to work: the role of depression. London: Mental Health Foundation.

Mental Health Strategies. 2012: 2011/12 National Survey of Investment in Adult Mental Health Services. Ref 1596-11 Report prepared for Department of Health.

Organisation for Economic Co-operation and Development (OECD). 2014: Mental health and work: United Kingdom. Paris: OECD Publishing.

Rost, K., Smith, J.L. and Dickinson, M. 2004: The effect of improving primary care depression management on employee absenteeism and productivity. A randomized trial. Medical Care 42, 1202-210.

Sainsbury Centre for Mental Health. 2007: Mental health at work: developing the business case. London: Sainsbury Centre for Mental Health.

Schoenbaum, M., Unutzer, J., McCaffrey, D., Duan, N., Sherbourne, C. and Wells, K.B. 2002: The effects of primary care depression treatment on patients' clinical status and employment. Health Services Research 37 (5), 1145-58.

Schoenbaum, M., Unutzer, J., Sherbourne, C., Duan, N., Rubenstein, L.V., Miranda, J., Meredith, L.S., Carney, M.F. and Wells, K. 2001: Cost-effectiveness of practice-initiated quality improvement for depression: results of a randomized controlled trial. Journal of American Medical Association 286, 1325-330.

Seymour, L. 2010: Common mental disorders at work. London: Sainsbury Centre for Mental Health.

Shiels, C., Gabbay, M. and Hillage, J. 2014: Factors associated with prevalence and types of 'may be fit' advice on fit notes: a cross-sectional primary care analysis. British Journal of General Practice 64, 137-43. 
Shiels, C., Hillage, J., Pollard, E. and Gabbay, M. 2013: National evaluation of the statement of fitness for work (Fit note): quantitative survey of fit notes. DWP research report no 841. London: The Stationery Office.

Simon, G.E., Katon, W., Rutter, C., Von Korff, M., Lin, E., Robinson, P., Bush, T., Walker, E.A., Ludman, E. and Russo, J. 1998: Impact of improved depression treatment in primary care on daily functioning and disability. Psychological Medicine 28, 693-701.
Vaez, M., Rylander, G., Nygren, A., Asberg, M. and Alexanderson, K. 2007: Sickness absence and disability pension in a cohort of employees on long-term sick leave due to psychiatric disorders in Sweden. Social Psychiatry and Psychiatric Epidemiology 42, 381-88.

Vos, T., Flaxman, A.D., Naghavi., M, et al. 2012: Years lived with disability (YLDs) for 1160 sequelae of 289 diseases and injuries 1990-2010: a systematic analysis for the global burden of disease study 2010. Lancet 380, 2163-196. 